Article

\title{
Novel Lycorine Derivatives as Anticancer Agents: Synthesis and In Vitro Biological Evaluation
}

\author{
Peng Wang ${ }^{1,2}$, Hui-Hui Yuan ${ }^{3}$, Xue Zhang ${ }^{1}$, Yun-Ping $\mathrm{Li}^{3}$, Lu-Qing Shang ${ }^{1, *}$ and Zheng Yin ${ }^{1, *}$ \\ 1 College of Pharmacy \& State Key Laboratory of Elemento-Organic Chemistry, Nankai University, \\ Tianjin 300071, China \\ 2 Department of Chemistry \& State Key Laboratory of Elemento-Organic Chemistry, \\ Nankai University, Tianjin 300071, China \\ 3 College of Life Sciences, Nankai University, Tianjin 300071, China \\ * Authors to whom correspondence should be addressed; E-Mails: shanglq@nankai.edu.cn (L.-Q.S.); \\ zheng_yin@nankai.edu.cn (Z.Y.).
}

Received: 29 January 2014; in revised form: 13 February 2014 / Accepted: 13 February 2014 /

Published: 21 February 2014

\begin{abstract}
Lycorine, which is the most abundant alkaloid isolated from the Amaryllidaceae family of plants, reportedly exhibits promising anticancer activities. Herein, a series of novel lycorine derivatives were synthesized and evaluated for their in vitro inhibitory activities against seven different cancer cell lines, including A549, HCT116, SK-OV-3, NCI-H460, K562, MCF-7 and HL-60. The results indicated that compounds bearing diverse amine substituents at the $\mathrm{C}-2$ position demonstrated good anticancer activities. The selectivity towards different cancer cell lines of the synthesized derivatives is discussed.
\end{abstract}

Keywords: lycorine derivatives; anticancer; synthesis

\section{Introduction}

Cancer is a proliferation disorder disease with apoptosis obstacles. It strikes more than one-third of the World's population and causes over $20 \%$ of all deaths [1]. The standard cancer treatment protocols include surgery, radiotherapy and chemotherapy. Unfortunately, chemotherapy is not effective in treating cancers associated with innate resistance to apoptosis and/or acquired resistance to drugs during treatment [2]. Discovery of novel effective anticancer medicines is therefore of great importance. As a source of drugs, natural products play an important role in drug discovery. Up to $80 \%$ 
of approved anticancer drugs over the time frame from 1981 to 2010 derived from natural products $[3,4]$. Plant species belonging to the Amaryllidaceae family have been well known for centuries to exhibit diverse medicinal properties. Many of these plants have been used historically for the treatment of cancer, since the oil of the daffodil Narcissus poeticus L. was used by Hippocrates of Kos [5]. The application of narcissus oil in cancer management continued during the middle ages in China, North Africa and Central America [6]. The major components isolated from the Amaryllidaceae plant family are alkaloids. Hundreds of alkaloids have been isolated and extensively studied for their pharmacological properties since 1877 [7]. Lycorine is one of the most common alkaloids within Amaryllidaceae family of plants and reportedly exhibits promising anticancer properties [7-12].

Lycorine has displayed multiple inhibitory properties towards various cancer cell lines, including lymphoma [7], carcinoma [8], multiple myeloma [9], melanoma [10], leukemia [7,11], human U373 glioblastoma, human A549 non-small-cell-lung cancer, human OE21 esophageal cancer and human Hs683 anaplastic oligodendroglioma cell lines [12]. Previous studies revealed that lycorine exhibited significantly higher antiproliferative activities in cancer cells than in normal cells, and it could be used to combat cancer cells which may or may not be sensitive to proapoptotic stimuli [12]. Further studies provided a mechanistic insight into its anticancer properties. Evdokimov et al. reported that lycorine exhibited cytostatic effects by targeting the actin cytoskeleton rather than by inducing apoptosis in cancer cells [13]. However, lycorine was found to induce apoptosis as well as arrest cell cycle of HL-60, KM3, K562, Hey1B cells in different phases [7,14-16]. Recently, the inhibitory effect on vasculogenic mimicry of melanoma cells [17] and suppression of neovascularization of ovarian cancer Hey1B cells by lycorine were reported [16]. Many factors thus seem involved in the anticancer properties of lycorine and a clear mechanism remains to be determined. Nevertheless, the anticancer properties of lycorine have drawn significant attention from chemists [5,6,11-13]. In the present study we report the synthesis of some novel lycorine derivatives and the evaluation of their in vitro anticancer activities.

\section{Results and Discussion}

\subsection{Chemistry}

The hydrochloride salt of lycorine (1) is commercially available, but its solubility is poor in most organic solvents. Therefore, 1, 2-diacetyllycorine (2) obtained from the acetylation of 1 with $\mathrm{Ac}_{2} \mathrm{O} / \mathrm{Py}$ was used as the key intermediate for further modifications.

Several derivatives were synthesized to investigate the influences of different substituents at the C-2 position. As outlined in the Scheme 1, the intermediate 3 was prepared via the selective deacetylation of the $\mathrm{C}-2$ hydroxyl group using conc. $\mathrm{HCl}$ in methanol since the $\mathrm{C}-2$ hydroxyl group is more reactive than the C-1 hydroxyl group [13]. The C-2 hydroxyl of $\mathbf{3}$ was then coupled with diverse acyl chlorides to afford derivatives 7a-e. Alternatively, the replacement of the $\mathrm{C}-2$ hydroxyl group by chloride using phosphorus oxychloride as halogenating agent provided 1-acetyl-2-chlorolycorine (4), which was further treated with sodium methoxide to produce 1,2- $\alpha$-epoxyllycorine (8). Compounds $9 \mathbf{a}-\mathbf{e}$ were obtained via the opening of the epoxide using the corresponding amines. The nucleophilic attack of the epoxide should occur from the least hindered side with high regioselectivity to afford $\mathbf{9 a}-\mathbf{e}$, according to a previous report [18]. This conclusion was confirmed using NOESY analysis. Taking $\mathbf{9 b}$ as an 
example, a correlation peak was found for $\mathrm{H} 1 \mathrm{~b} / \mathrm{H} 1$, but no correlation between $\mathrm{H} 1 \mathrm{~b} / \mathrm{H} 2$ was observed (Figure 1). Oxidation of the double bond in D-ring with $m$-CPBA led to derivatives 5 and $\mathbf{6}$.

Scheme 1. Synthesis of lycorine derivatives.

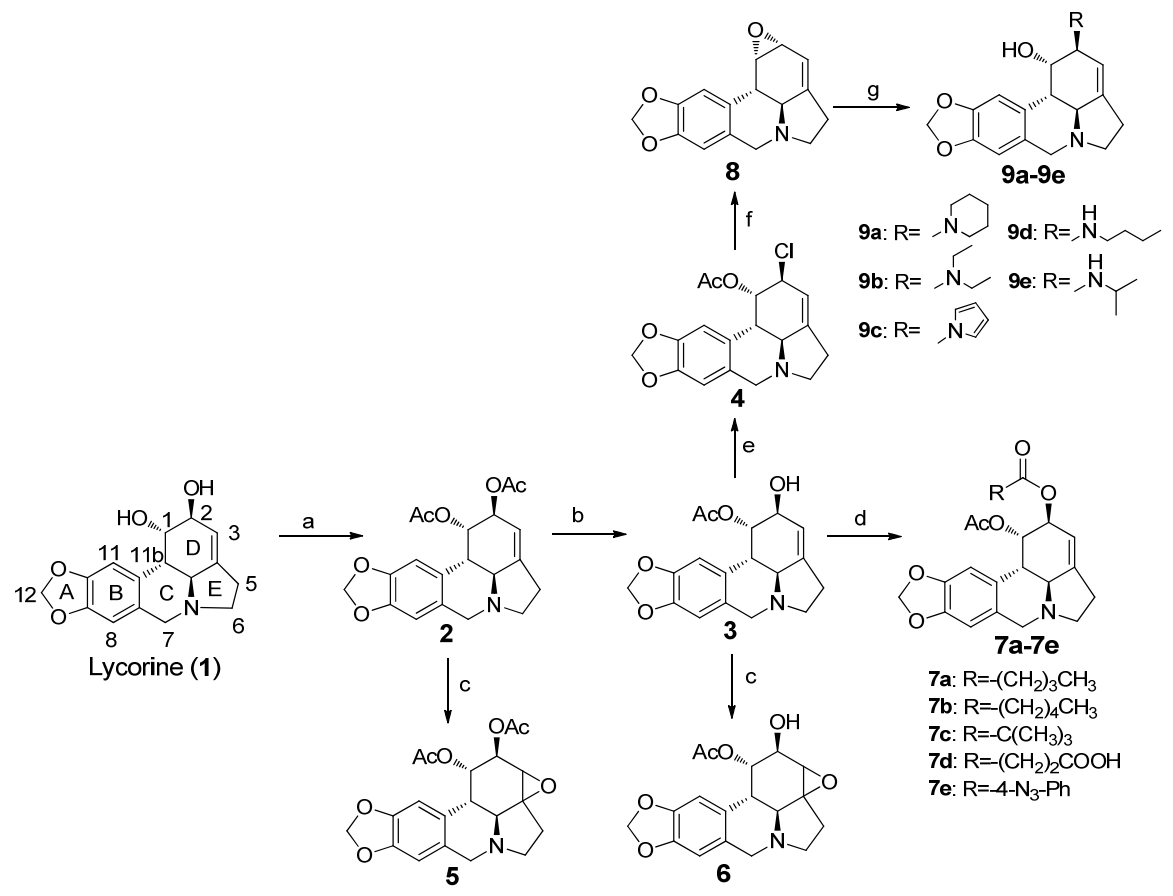

Reagents and conditions: (a) $\mathrm{Ac}_{2} \mathrm{O}$, Py; (b) $\mathrm{HCl}, \mathrm{MeOH}$; (c) $m$-CPBA, DCM; (d) RCOCl, Py, DCM; (e) $\mathrm{POCl}_{3}, \mathrm{NaCl}, \mathrm{HCl}$; (f) $\mathrm{CH}_{3} \mathrm{ONa}, \mathrm{MeOH}$; (g) $\mathrm{RNH}_{2}$ or $\mathrm{R}_{2} \mathrm{NH}$.

Figure 1. NOESY analysis of $9 e$.

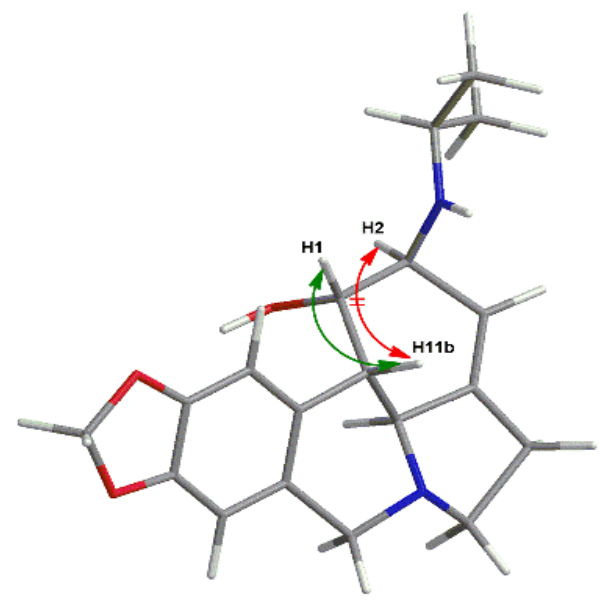

\subsection{Biological Activity Results}

In vitro anticancer activities of the synthesized lycorine derivatives were evaluated using the MTT colorimetric assay against a panel of seven human cancer cell lines [19], including non-small-cell-lung cancer (A549), colon carcinoma (HCT116), ovarian carcinoma (SK-OV-3), large-cell-lung cancer (NCI-H460), human myelogenous leukemia (K562), promyelocytic leukemia (HL-60) and human breast adenocarcinoma (MCF-7) cell lines. The results were expressed as $\mathrm{IC}_{50}$ for the inhibitory activities and are summarized in Table 1. 
Table 1. In vitro anticancer activities of lycorine derivatives.

\begin{tabular}{|c|c|c|c|c|c|c|c|c|}
\hline \multirow{2}{*}{ No. } & \multirow{2}{*}{$\operatorname{cLog} \mathrm{P}^{a}$} & \multicolumn{7}{|c|}{$\mathrm{IC}_{50} / \boldsymbol{\mu} \mathrm{M}^{b}$} \\
\hline & & A549 & HCT116 & SK-OV-3 & NCI-H460 & K562 & MCF-7 & HL-60 \\
\hline 1 & 0.39 & $6.5 \pm 0.3$ & $3.0 \pm 0.2$ & $3.0 \pm 0.3$ & $3.3 \pm 0.3$ & $7.5 \pm 0.5$ & $3.9 \pm 0.2$ & $4.1 \pm 0.1$ \\
\hline 2 & 2.16 & $20.6 \pm 0.8$ & $>20$ & $16.4 \pm 0.5$ & $>20$ & $>20$ & $>20$ & $>20$ \\
\hline 3 & 1.26 & $>20$ & $9.4 \pm 0.5$ & $18.2 \pm 0.2$ & $10.6 \pm 0.4$ & $18.6 \pm 0.3$ & $19.5 \pm 0.2$ & $>20$ \\
\hline 4 & 2.73 & $>20$ & $>20$ & $>20$ & $>20$ & $>20$ & $>20$ & $>20$ \\
\hline 5 & 1.54 & $>20$ & $9.8 \pm 1.0$ & $>20$ & $>20$ & $>20$ & $>20$ & $>20$ \\
\hline 6 & 0.69 & $>20$ & $>20$ & $>20$ & $>20$ & $>20$ & $>20$ & $>20$ \\
\hline $7 a$ & 3.74 & $>20$ & $10.2 \pm 0.6$ & $>20$ & $17.8 \pm 0.3$ & $>20$ & $18.1 \pm 0.3$ & $>20$ \\
\hline $7 b$ & 4.27 & $19.2 \pm 0.5$ & $13.6 \pm 1.3$ & $10.1 \pm 0.3$ & $13.1 \pm 0.4$ & $>20$ & $17.8 \pm 0.5$ & $>20$ \\
\hline $7 c$ & 3.39 & $>20$ & $13.5 \pm 0.6$ & $16.7 \pm 0.5$ & $14.3 \pm 0.4$ & $20.9 \pm 0.5$ & $19.1 \pm 0.3$ & $>20$ \\
\hline $7 d$ & -0.35 & $>20$ & $>20$ & $>20$ & $>20$ & $>20$ & $>20$ & $>20$ \\
\hline $7 e$ & 4.70 & $18.7 \pm 1.1$ & $13.8 \pm 0.2$ & $17.2 \pm 0.6$ & $19.7 \pm 0.6$ & $18.5 \pm 0.3$ & $>20$ & $>20$ \\
\hline 8 & 1.35 & $6.2 \pm 0.4$ & $3.9 \pm 0.3$ & $3.3 \pm 0.3$ & $2.7 \pm 0.1$ & $3.4 \pm 0.2$ & $4.5 \pm 0.2$ & $2.7 \pm 0.3$ \\
\hline $9 a$ & 2.48 & $14.2 \pm 0.5$ & $7.9 \pm 0.3$ & $4.5 \pm 0.3$ & $9.9 \pm 0.4$ & $>20$ & $>20$ & $8.3 \pm 0.2$ \\
\hline $9 b$ & 2.42 & $15.2 \pm 0.6$ & $6.4 \pm 0.2$ & $16.8 \pm 0.4$ & $8.0 \pm 0.4$ & $12.8 \pm 0.5$ & $5.2 \pm 0.3$ & $>20$ \\
\hline $9 c$ & 2.14 & $>20$ & $>20$ & $15.5 \pm 0.3$ & $8.1 \pm 0.7$ & $12.0 \pm 0.8$ & $20.9 \pm 0.6$ & $9.1 \pm 0.5$ \\
\hline 9d & 2.36 & $16.3 \pm 1.3$ & $10.1 \pm 0.1$ & $12.6 \pm 0.7$ & $6.5 \pm 0.2$ & $6.1 \pm 0.3$ & $>20$ & $9.7 \pm 0.2$ \\
\hline $9 e$ & 1.61 & $>20$ & $20.5 \pm 0.7$ & $13.4 \pm 0.2$ & $19.8 \pm 0.3$ & $>20$ & $19.7 \pm 0.5$ & $>20$ \\
\hline
\end{tabular}

${ }^{a}$ : cLog P was calculated using ChemBioDraw; ${ }^{b}$ : Values are the mean of at least three independent experiments.

Evidente et al. reported that epoxide $\mathbf{8}$ demonstrated anticancer activities comparable with lycorine against the A549, OE21, Hs683, U373, SKMEL and B16F10 cell lines [12]. In our study, 8 also presented excellent potency against all tested cell lines. However, compared with $\mathbf{2}$ and $\mathbf{3}$, epoxides $\mathbf{5}$ and $\mathbf{6}$ derived from $\mathbf{2}$ and $\mathbf{3}$, respectively, showed much reduced activity $(>20 \mu \mathrm{M})$ against almost all seven cell lines. The results indicated that an epoxide ring at $\mathrm{C} 3-\mathrm{C} 4$ didn't benefit the anticancer activities and the presence of the double bond of A-ring appeared to be essential for the potency.

Esters $7 \mathbf{a}-\mathbf{e}$ were synthesized to investigate the influence of modifications of the C-2 hydroxyl group. In general, $\mathbf{7 a}, \mathbf{7 b}$, and $\mathbf{7} \mathbf{c}$ exhibited moderate potency against all of tested cell lines, except K562 and HL-60, while 7e demonstrated moderate potency against all of the tested cell lines except MCF-7 and HL-60. Interesting selectivity was observed with ester substituents as well. Generally, esterification of the C-2 hydroxyl group with aliphatic or aromatic acids slightly increased the activities for the A549 and SK-OV-3 cell lines, but decreased the activities for HCT116, NCI-H460, K562 and MCF-7 at different levels (2, 7a, 7b, 7c, 7e vs. 3). For the HCT116 and NCI-H460 cell lines, both the aliphatic $(\mathbf{2}, \mathbf{7} \mathbf{a}, \mathbf{7 b}, \mathbf{7} \mathbf{c})$ and the aromatic substituent $(\mathbf{7 e})$ at the $\mathrm{C}-2$ position appeared to be tolerated well, but for the K562 cell line, an aromatic substituent (compound 7e) exhibited better activities than linear aliphatic substituents $(\mathbf{2}, \mathbf{7 a}, \mathbf{7 b})$. However, the results were the opposite for the MCF-7 cell line. It is noteworthy that $\mathbf{7 d}$ with a carboxylic acid terminus lost its activity against all of the tested cell lines. The decrease in activities for $\mathbf{7 d}$ might result from the ionic nature of the carboxylate group and the significant change of cLog P. In a previous report [20], cell permeability was an important determinant for anticancer activities of lycorine derivatives. It is likely that this observation reflects the difficulty of cell penetration for highly hydrophilic lycorine derivatives like 7d. In another variation, the hydroxyl group at the C-2 position was replaced by a chlorine to afford 4. 
This replacement led to the dramatic decrease in potency against all of the tested cell lines $(>20 \mu \mathrm{M})$.

To further examine the influence of substituents at the C-2 position, a variety of amines (alkylamine, piperidine, pyrrole) were introduced to yield $\mathbf{9 a}-\mathbf{e}$ via the opening of the epoxide of $\mathbf{8}$. Overall, these derivatives exhibited higher potency than esters 7a-e. From the perspective of hydrogen-bond acceptor/donor, tertiary amino substituents at the C-2 position of compounds 9a-c could be an acceptor, while the secondary amino substituents of compounds $\mathbf{9 d}-\mathbf{e}$ could be both an acceptor and a donor. No clearly difference was observed between the activities of $9 \mathbf{a}-\mathbf{c}$ and $9 \mathbf{d}-\mathbf{e}$ towards all of tested cell lines. Thus, a hydrogen donor at this position might be less important for the anticancer activities than a hydrogen acceptor. Lycorine exhibited similar good activities towards all of tested cell lines, while amine substituents at the C-2 position improved the selectivity to different cancer cell lines. Aliphatic substituents (compounds 9a, 9b, 9d) exhibited better activities than aromatic substituent (9c) for A549 and HCT116 cell lines. For SK-OV-3, 9a $\left(\mathrm{IC}_{50}=4.5 \mu \mathrm{M}\right)$ represented comparable activity with lycorine $\left(\mathrm{IC}_{50}=3.0 \mu \mathrm{M}\right)$. The compound with a linear aliphatic substituent 9d provided the best activities for NCI-H460 $\left(\mathrm{IC}_{50}=6.5 \mu \mathrm{M}\right)$ and $\mathrm{K} 562\left(\mathrm{IC}_{50}=6.1 \mu \mathrm{M}\right.$, which is better than that of lycorine, $\mathrm{IC}_{50}=7.5 \mu \mathrm{M}$ ) while the bulky aliphatic substituent derivative $9 \mathbf{b}$ demonstrated the best potency against $\mathrm{HCT} 116\left(\mathrm{IC}_{50}=6.4 \mu \mathrm{M}\right)$ and $\mathrm{MCF}-7\left(\mathrm{IC}_{50}=5.2 \mu \mathrm{M}\right)$.

\section{Experimental}

\subsection{General}

All solvents were treated by standard techniques before use. Lycorine hydrochloride (purity $>98 \%$ ) was purchased from Baoji Guokang Biotechnology Co. Ltd (Baoji, China). Cell culture medium, fetal bovine serum, and trypsin were purchased from Thermo Scientific (Waltham, MA, USA). All reagents were purchased from the commercial suppliers and were used without further purification. ${ }^{1} \mathrm{H}-$ and ${ }^{13} \mathrm{C}-\mathrm{NMR}$ spectra were recorded on a Bruker AVANCE-400 (400 MHz) NMR spectrometer (Billerica, MA, USA) $\left({ }^{1} \mathrm{H}, 400 \mathrm{MHz} ;{ }^{13} \mathrm{C}, 100 \mathrm{MHz}\right)$ in $\mathrm{CDCl}_{3}, \mathrm{CD}_{3} \mathrm{OD}$ using TMS as an internal standard. Chemical shifts are reported in parts per million $\delta$ in ppm and coupling constants are provided in hertz (Hz). ESI-MS spectra were recorded on a Shimadzu LCMS-2020 (Shimadzu, Kyoto, Japan). HRMS were recorded on a Varian 7.0 T high resolution ESI-FTICR mass spectrometer (Palo Alto, CA, USA). Silica gel TLC (GF254) was used to monitor the reactions, which were visualized under UV light at $254 \mathrm{~nm}$ and $365 \mathrm{~nm}$. Flash chromatography was performed using silica gel (200-300 mesh).

\subsection{Cell Lines}

The cell lines used in this study were purchased from the Shanghai Cell Bank, Chinese Academy of Medical Sciences (Shanghai, China). All cell lines were maintained in a humidified atmosphere containing 5\% $\mathrm{CO}_{2}$ at $37{ }^{\circ} \mathrm{C}$. All the cancer cells were cultured in RPMI-1640 medium supplemented with $10 \% \mathrm{FBS}, 100 \mathrm{U} / \mathrm{mL}$ penicillin, and $100 \mu \mathrm{g} / \mathrm{mL}$ streptomycin. The cells were seeded into 96 well plates in $100 \mu \mathrm{L}$ at plating density of $2 \times 10^{3}$ cells per well. After incubation for $24 \mathrm{~h}$, the cells were allowed to attach. The cells were treated with different concentrations of compounds and then incubated for $72 \mathrm{~h}$ under the same conditions. Compounds were dissolved in DMSO at stock concentrations of $20 \mathrm{mM}$. 


\subsection{In Vitro Growth Inhibition Assay}

The concentrations which were used to calculate the $\mathrm{IC}_{50}$ values were: $100,50,25,12.5,6.25$, $3.125,1.56,0.78 \mu \mathrm{M}$. The stock solutions were diluted with complete medium before use. Untreated cells were used as controls. The cells were then treated for $72 \mathrm{~h}$ with varying concentrations of compounds. $20 \mu \mathrm{L}$ of 3-(4,5 dimethylthia-zol-2-yl)-2,5-diphenyl-tetrazoliumbromide (MTT, Sigma, St. Louis, MO, USA) solution ( $5 \mathrm{mg} / \mathrm{mL}$ in PBS) was added to each well and incubated for $4 \mathrm{~h}$ at $37{ }^{\circ} \mathrm{C}$. After the removal of the medium, MTT formazan was dissolved in $150 \mu \mathrm{L}$ DMSO and monitored using a microplate reader at a wavelength of $570 \mathrm{~nm}$. All experiments were performed in triplicate wells and were repeated at least three times.

\subsection{Chemistry}

(1S, 2S,3a $\left.a^{1} S, 12 b S\right)-2,3 a^{1}, 4,5,7,12 b$-Hexahydro-1H-[1,3]dioxolo[4,5-j]pyrrolo[3,2,1-de]phenanthridine1,2-diyl diacetate (1,2-diacetyllycorine, 2). To a solution of lycorine $(3.17 \mathrm{~g}, 11 \mathrm{mmol})$ in pyridine $(8.0 \mathrm{~mL})$ was added acetic anhydride $(9.5 \mathrm{~mL})$ and the solution was stirred at $50{ }^{\circ} \mathrm{C}$ for $12 \mathrm{~h}$. Subsequently methanol $(25 \mathrm{~mL})$ was added. The mixture was stirred at r.t. for $3 \mathrm{~h}$. The solvent was removed under reduced pressure followed by the addition of DCM $(50 \mathrm{~mL})$ and water $(40 \mathrm{~mL})$. The organic layer was washed with aqueous $\mathrm{NaHCO}_{3}$ solution and brine, respectively, and dried over anhydrous $\mathrm{Na}_{2} \mathrm{SO}_{4}$. After filtration, the filtrate was concentrated and the crude residue was purified using a silica gel chromatography and was eluted with $\mathrm{DCM} / \mathrm{EtOAc} / \mathrm{CH}_{3} \mathrm{OH}(10: 10: 1)$ to give 2 as a white solid (3.78 g, 93.3\%). ${ }^{1} \mathrm{H}-\mathrm{NMR}\left(400 \mathrm{MHz}, \mathrm{CDCl}_{3}\right) \delta: 6.75(\mathrm{~s}, 1 \mathrm{H}), 6.57(\mathrm{~s}, 1 \mathrm{H}), 5.91(\mathrm{~s}, 2 \mathrm{H})$, $5.74(\mathrm{~s}, 1 \mathrm{H}), 5.53(\mathrm{~s}, 1 \mathrm{H}), 5.26(\mathrm{~s}, 1 \mathrm{H}), 4.16(\mathrm{~d}, J=14.0 \mathrm{~Hz}, 1 \mathrm{H}), 3.53(\mathrm{~d}, J=14.0 \mathrm{~Hz}, 1 \mathrm{H}), 3.37(\mathrm{~m}$, $1 \mathrm{H}), 2.86(\mathrm{~d}, J=10.0 \mathrm{~Hz}, 1 \mathrm{H}), 2.77(\mathrm{~d}, J=10.4 \mathrm{~Hz}, 1 \mathrm{H}), 2.65(\mathrm{~m}, 2 \mathrm{H}), 2.40(\mathrm{~m}, 1 \mathrm{H}), 2.08(\mathrm{~s}, 3 \mathrm{H})$, 1.95 (s, 3H); ${ }^{13} \mathrm{C}-\mathrm{NMR}\left(100 \mathrm{MHz}, \mathrm{CDCl}_{3}\right) \delta: 170.0,169.8,146.4,146.2,129.4,126.5,113.8,107.3$, 105.0, 101.0, 70.9, 69.2, 61.2, 56.9, 53.6, 40.5, 28.7, 21.2, 21.0; ESI-MS: $m / z 372[\mathrm{M}+\mathrm{H}]^{+}$; HRMS: $\mathrm{m} / z$ calcd. for $\mathrm{C}_{20} \mathrm{H}_{22} \mathrm{NO}_{6} 372.1442[\mathrm{M}+\mathrm{H}]^{+}$; found: 372.1445 .

(1S,2S,3 $\left.a^{1} S, 12 b S\right)$-2-Hydroxy-2,3a $a^{1}$ 4, 5, 7,12b-hexahydro-1H-[1,3]dioxolo[4,5-j]pyrrolo[3,2,1-de]phenanthridin-1-yl acetate (1-acetyllycorine, 3). To a solution of 2 (0.96 g, $2.58 \mathrm{mmol})$ in methanol $(100 \mathrm{~mL})$ was added conc. $\mathrm{HCl}(20 \mathrm{~mL})$, and the solution was stirred at $55{ }^{\circ} \mathrm{C}$ for $1 \mathrm{~h}$. The mixture was made alkaline $(\mathrm{pH}=8)$ with aqueous $\mathrm{NaHCO}_{3}$ solution, followed by the addition of DCM $(25 \mathrm{~mL})$. The organic layer was washed with brine $(25 \mathrm{~mL})$, dried over anhydrous $\mathrm{Na}_{2} \mathrm{SO}_{4}$ and filtered. The filtrate was concentrated under reduced pressure and the crude residue was purified using silica gel chromatography and was eluted with $\mathrm{EtOAc} / \mathrm{CH}_{3} \mathrm{OH}(10: 1)$ to give 3 as a white solid $(0.50 \mathrm{~g}$, 58.9\%). ${ }^{1} \mathrm{H}-\mathrm{NMR}\left(400 \mathrm{MHz}, \mathrm{CDCl}_{3}\right) \delta: 6.66(\mathrm{~s}, 1 \mathrm{H}), 6.57(\mathrm{~s}, 1 \mathrm{H}), 5.92(\mathrm{~s}, 2 \mathrm{H}), 5.61(\mathrm{~s}, 1 \mathrm{H}), 5.54(\mathrm{~s}$, $1 \mathrm{H}), 4.18(\mathrm{~s}, 1 \mathrm{H}), 4.25(\mathrm{~d}, J=14.4 \mathrm{~Hz}, 1 \mathrm{H}), 3.50(\mathrm{~d}, J=14.0 \mathrm{~Hz}, 1 \mathrm{H}), 3.35(\mathrm{~m}, 1 \mathrm{H}), 2.85(\mathrm{~d}, J=10.4 \mathrm{~Hz}$, 1H), $2.74(\mathrm{~d}, J=10.4 \mathrm{~Hz}, 1 \mathrm{H}), 2.63(\mathrm{~m}, 2 \mathrm{H}), 2.37(\mathrm{~m}, 1 \mathrm{H}), 1.94(\mathrm{~s}, 3 \mathrm{H}) ;{ }^{13} \mathrm{C}-\mathrm{NMR}\left(100 \mathrm{MHz}, \mathrm{CDCl}_{3}\right)$ $\delta: 170.8,146.4,146.2,143.3,129.1,127.0,117.6,107.3,104.9,101.0,72.7,69.2,61.6,56.8,53.7$, 39.0, 28.5, 21.1; ESI-MS: $m / z 330[\mathrm{M}+\mathrm{H}]^{+}$; HRMS: $m / z$ calcd. for $\mathrm{C}_{18} \mathrm{H}_{20} \mathrm{NO}_{5} 330.1341[\mathrm{M}+\mathrm{H}]^{+}$; found: 330.1344 . 
(1S,2S,3a $\left.a^{1} S, 12 b S\right)$-2-Chloro-2,3a $, 4,5,7,12 b$-hexahydro-1H-[1,3]dioxolo[4,5-j]pyrrolo[3,2,1-de]-phenanthridin-1-yl acetate (1-acetyl-2-chlorollycorine, 4). To a solution of $\mathrm{NaCl}(5 \mathrm{mg}, 0.1 \mathrm{mmol})$ in phosphorus oxychloride $(2 \mathrm{~mL})$ was added $3(156 \mathrm{mg}, 0.47 \mathrm{mmol})$, and the mixture was stirred at $30{ }^{\circ} \mathrm{C}$ for $1 \mathrm{~h}$, followed by the addition of conc. $\mathrm{HCl}$ solution $(0.1 \mathrm{~mL})$. After stirred at $30{ }^{\circ} \mathrm{C}$ for another $2 \mathrm{~h}$, the mixture was poured into ice water, made alkaline $(\mathrm{pH}=8)$ with aqueous ammonia solution and was extracted with DCM $(25 \mathrm{~mL})$. The organic layer was dried over anhydrous $\mathrm{Na}_{2} \mathrm{SO}_{4}$ and filtered. The filtrate was concentrated under reduced pressure and the crude residue was purified using silica gel chromatography and eluted with EtOAc/ $\mathrm{CH}_{3} \mathrm{OH}(10: 1)$ to give 4 as a white solid (132 mg, 80.9\%). ${ }^{1} \mathrm{H}-\mathrm{NMR}\left(400 \mathrm{MHz}, \mathrm{CDCl}_{3}\right) \delta: 6.72(\mathrm{~s}, 1 \mathrm{H}), 6.58(\mathrm{~s}, 1 \mathrm{H}), 5.92(\mathrm{~s}, 2 \mathrm{H}), 5.88(\mathrm{~s}, 1 \mathrm{H}), 5.55$ $(\mathrm{s}, 1 \mathrm{H}), 4.06(\mathrm{~s}, 1 \mathrm{H}), 4.15(\mathrm{~d}, J=14.4 \mathrm{~Hz}, 1 \mathrm{H}), 3.55(\mathrm{~d}, J=14.0 \mathrm{~Hz}, 1 \mathrm{H}), 3.37(\mathrm{~m}, 1 \mathrm{H}), 3.18(\mathrm{~d}$, $J=10.4 \mathrm{~Hz}, 1 \mathrm{H}), 2.87(\mathrm{~d}, J=10.0 \mathrm{~Hz}, 1 \mathrm{H}), 2.66(\mathrm{~m}, 2 \mathrm{H}), 2.42(\mathrm{~m}, 1 \mathrm{H}), 1.96(\mathrm{~s}, 3 \mathrm{H}) ;{ }^{13} \mathrm{C}-\mathrm{NMR}(100$ $\left.\mathrm{MHz}, \mathrm{CDCl}_{3}\right) \delta: 170.2,146.5,146.4,144.0,129.4,126.5,116.2,107.4,104.8,101.0,71.8,61.3,56.9$, 56.7, 53.6, 38.4, 28.7, 20.9; ESI-MS: $m / z 348$ [M + H] ${ }^{+}$; HRMS: $m / z$ calcd. for $\mathrm{C}_{18} \mathrm{H}_{19} \mathrm{ClNO}_{4} 348.0997$ $[\mathrm{M}+\mathrm{H}]^{+}$; found: 348.0990 .

(1a $\left.a^{1} S, 10 b S, 11 S, 12 R\right)-1 a^{1}, 2,3,5,10 b, 11,12,12 a-O c t a h y d r o-[1,3]$ dioxolo[4,5-j]oxireno[2,3-c]pyrrolo[3,2,1-de]phenanthridine-11,12-diyl diacetate (1,2-diacetyl-3,4-epoxyllycorine, 5). To a solution of 2 (371 mg, $1 \mathrm{mmol}$ ) in $5 \mathrm{~mL}$ DCM was added $m$-CPBA (260 mg, $1.5 \mathrm{mmol}$ ), and the mixture was stirred at r.t. for $1 \mathrm{~h}$. After solvent evaporation, the crude residue was purified using a silica gel chromatography eluted with $\mathrm{DCM} / \mathrm{CH}_{3} \mathrm{OH}$ (3:1) to give 5 as a white solid (324 mg, 83.7\%). ${ }^{1} \mathrm{H}-\mathrm{NMR}$ $\left(400 \mathrm{MHz}, \mathrm{CD}_{3} \mathrm{OD}\right) \delta: 6.76(\mathrm{~s}, 1 \mathrm{H}), 6.59(\mathrm{~s}, 1 \mathrm{H}), 5.86(\mathrm{~s}, 2 \mathrm{H}), 5.82(\mathrm{~s}, 1 \mathrm{H}), 5.67(\mathrm{~s}, 1 \mathrm{H}), 5.20(\mathrm{~s}, 1 \mathrm{H})$, $4.74(\mathrm{~d}, J=15.2 \mathrm{~Hz}, 1 \mathrm{H}), 4.48(\mathrm{~d}, J=14.8 \mathrm{~Hz}, 1 \mathrm{H}), 4.13(\mathrm{~d}, J=11.6 \mathrm{~Hz}, 1 \mathrm{H}), 3.83(\mathrm{~m}, 1 \mathrm{H}), 3.49$ (d, $J=11.6 \mathrm{~Hz}, 1 \mathrm{H}), 2.96(\mathrm{~m}, 1 \mathrm{H}), 2.79(\mathrm{~m}, 1 \mathrm{H}), 1.99(\mathrm{~s}, 3 \mathrm{H}), 1.83(\mathrm{~s}, 3 \mathrm{H}) ;{ }^{13} \mathrm{C}-\mathrm{NMR}(100 \mathrm{MHz}$, $\left.\mathrm{CD}_{3} \mathrm{OD}\right) \delta: 171.3,171.2,149.0,148.8,141.3,125.9,125.7,119.0,108.6,106.0,103.0,72.5,70.7$, 70.0, 69.0, 68.6, 35.7, 27.6, 20.9, 20.6; ESI-MS: $m / z 388[\mathrm{M}+\mathrm{H}]^{+}$; HRMS: $m / z$ calcd. for $\mathrm{C}_{20} \mathrm{H}_{22} \mathrm{NO}_{7}$ $388.1391[\mathrm{M}+\mathrm{H}]^{+}$; found: 388.1386 .

(1a $\left.{ }^{1} S, 10 b S, 11 S, 12 R\right)$-12-Hydroxy-1a $a^{1}, 2,3,5,10 b, 11,12,12$-octahydro-[1,3]dioxolo[4,5-j]oxireno[2,3c]pyrrolo[3,2,1-de]phenanthridin-11-yl acetate (1-acetyl-3,4-epoxyllycorine, 6). To a solution of $\mathbf{3}$ (200 mg, $0.61 \mathrm{mmol})$ in DCM (4 mL) was added $m$-CPBA (210 mg, $1.22 \mathrm{mmol})$, and the mixture was stirred at r.t. for $1 \mathrm{~h}$. After solvent evaporation, the crude residue was purified using silica gel chromatography and eluted with $\mathrm{DCM} / \mathrm{CH}_{3} \mathrm{OH}$ (3:1) to give 6 as a white solid (153 $\mathrm{mg}, 72.7 \%$ ). ${ }^{1} \mathrm{H}-\mathrm{NMR}\left(400 \mathrm{MHz}, \mathrm{CD}_{3} \mathrm{OD}\right) \delta: 6.78(\mathrm{~s}, 1 \mathrm{H}), 6.63(\mathrm{~s}, 1 \mathrm{H}), 5.90(\mathrm{~s}, 2 \mathrm{H}), 5.78(\mathrm{~s}, 1 \mathrm{H}), 5.73(\mathrm{~s}, 1 \mathrm{H})$, $4.77(\mathrm{~d}, J=15.2 \mathrm{~Hz}, 1 \mathrm{H}), 4.52(\mathrm{~d}, J=15.2 \mathrm{~Hz}, 1 \mathrm{H}), 4.13(\mathrm{~s}, 1 \mathrm{H}), 4.12(\mathrm{~d}, J=10.4 \mathrm{~Hz}, 1 \mathrm{H}), 3.84(\mathrm{~m}$, 2H), $3.52(\mathrm{~d}, J=11.2 \mathrm{~Hz}, 1 \mathrm{H}), 2.98(\mathrm{~m}, 1 \mathrm{H}), 2.82(\mathrm{~m}, 1 \mathrm{H}), 1.86(\mathrm{~s}, 3 \mathrm{H}) ;{ }^{13} \mathrm{C}-\mathrm{NMR}(100 \mathrm{MHz}$, $\left.\mathrm{CD}_{3} \mathrm{OD}\right) \delta: 171.7,148.9,148.6,137.9,126.7,125.6,122.7,108.5,105.6,102.9,72.8,72.6,69.3,69.0$, 68.6, 34.4, 27.37, 20.7; ESI-MS: $m / z 346[\mathrm{M}+\mathrm{H}]^{+}$; HRMS: $m / z$ calcd. for $\mathrm{C}_{18} \mathrm{H}_{20} \mathrm{NO}_{6} 346.1258[\mathrm{M}+\mathrm{H}]^{+}$; found: 346.1257 .

\subsubsection{General Procedure for 7}

To a solution of 3 (1 equiv.) in anhydrous pyridine $(2 \mathrm{~mL})$ was added acyl chloride or anhydride (1.2 equiv.) in anhydrous DCM $(5 \mathrm{~mL})$ over $15 \mathrm{~min}$ at $0{ }^{\circ} \mathrm{C}$, and the solution was stirred at $0{ }^{\circ} \mathrm{C}$ for 
another $3 \mathrm{~h}$. Subsequently, DCM and water were added, and the organic layer was washed using aqueous $\mathrm{NaHCO}_{3}$ solution and brine, dried over anhydrous $\mathrm{Na}_{2} \mathrm{SO}_{4}$ and filtered. The filtrate was concentrated under reduced pressure and the crude residue was purified using a silica gel chromatography to give $7 \mathbf{a}-\mathbf{e}$.

(1S,2S,3a $\left.a^{1} S, 12 b S\right)$-1-Acetoxy-2,3a 1 4,5,7,12b-hexahydro-1H-[1,3]dioxolo[4,5-j]pyrrolo[3,2,1-de]phenanthridin-2-yl pentanoate (1-acetyl-2-valeryllycorine, 7a). Following the previously described procedure, $95 \mathrm{mg}(0.28 \mathrm{mmol})$ of $\mathbf{3}$ gave $7 \mathbf{a}$ as a pale solid $(163 \mathrm{mg}, 64.7 \%) .{ }^{1} \mathrm{H}-\mathrm{NMR}$ $\left(400 \mathrm{MHz}, \mathrm{CDCl}_{3}\right) \delta: 6.75(\mathrm{~s}, 1 \mathrm{H}), 6.57(\mathrm{~s}, 1 \mathrm{H}), 5.92(\mathrm{~s}, 2 \mathrm{H}), 5.73(\mathrm{~s}, 1 \mathrm{H}), 5.53(\mathrm{~s}, 1 \mathrm{H}), 5.26(\mathrm{~s}, 1 \mathrm{H})$, $4.16(\mathrm{~d}, J=14.1 \mathrm{~Hz}, 1 \mathrm{H}), 3.55(\mathrm{~d}, J=14.0 \mathrm{~Hz}, 1 \mathrm{H}), 3.38(\mathrm{dt}, J=9.1,4.7 \mathrm{~Hz}, 1 \mathrm{H}), 2.89$ (d, $J=10.5 \mathrm{~Hz}$, $1 \mathrm{H}), 2.81(\mathrm{~d}, J=10.5 \mathrm{~Hz}, 1 \mathrm{H}), 2.66(\mathrm{~s}, 2 \mathrm{H}), 2.43(\mathrm{dd}, J=17.5,8.7 \mathrm{~Hz}, 1 \mathrm{H}), 2.33(\mathrm{td}, J=7.4,3.3 \mathrm{~Hz}$, 2H), $1.95(\mathrm{~s}, 3 \mathrm{H}), 1.61(\mathrm{dd}, J=15.2,7.6 \mathrm{~Hz}, 2 \mathrm{H}), 1.36(\mathrm{dd}, J=15.0,7.4 \mathrm{~Hz}, 3 \mathrm{H}), 0.91(\mathrm{t}, J=7.3 \mathrm{~Hz}$, $3 \mathrm{H}) ;{ }^{13} \mathrm{C}-\mathrm{NMR}\left(100 \mathrm{MHz}, \mathrm{CDCl}_{3}\right) \delta: 172.5,170.0,146.5,146.3,145.8,129.3,126.6,114.0,107.3,105.1$, 101.0, 70.6, 69.2, 61.2, 56.7, 53.6, 40.4, 34.1, 28.7, 26.9, 22.2, 20.9, 13.7; ESI-MS: $m / z 414[\mathrm{M}+\mathrm{H}]^{+}$; HRMS: $m / z$ calcd. for $\mathrm{C}_{23} \mathrm{H}_{28} \mathrm{NO}_{6} 414.1917[\mathrm{M}+\mathrm{H}]^{+}$; found: 414.1905 .

(1S,2S,3a $S, 12 b S)$-1-Acetoxy-2,3a $, 4,5,7,12 b$-hexahydro-1H-[1,3]dioxolo[4,5-j]pyrrolo[3,2,1-de]phenanthridin-2-yl hexanoate (1-acetyl-2-hexanoyllycorine, 7b). Following the previously described procedure, $95 \mathrm{mg}(0.28 \mathrm{mmol})$ of $\mathbf{3}$ gave $\mathbf{7 b}$ as a white solid $(99 \mathrm{mg}, 75.0 \%)$. ${ }^{1} \mathrm{H}-\mathrm{NMR}$ $\left(400 \mathrm{MHz}, \mathrm{CDCl}_{3}\right) \delta: 6.71(\mathrm{~s}, 1 \mathrm{H}), 6.54(\mathrm{~s}, 1 \mathrm{H}), 5.88(\mathrm{~s}, 2 \mathrm{H}), 5.70(\mathrm{~s}, 1 \mathrm{H}), 5.49(\mathrm{~s}, 1 \mathrm{H}), 5.23(\mathrm{~s}, 1 \mathrm{H})$, $4.13(\mathrm{~d}, J=14.0 \mathrm{~Hz}, 1 \mathrm{H}), 3.50(\mathrm{~d}, J=14.0 \mathrm{~Hz}, 1 \mathrm{H}), 3.34(\mathrm{~m}, 1 \mathrm{H}), 2.85(\mathrm{~d}, J=10.0 \mathrm{~Hz}, 1 \mathrm{H}), 2.75(\mathrm{~d}$, $J=10.4 \mathrm{~Hz}, 1 \mathrm{H}), 2.62(\mathrm{~m}, 2 \mathrm{H}), 2.37(\mathrm{~m}, 1 \mathrm{H}), 2.29(\mathrm{~m}, 2 \mathrm{H}), 1.92(\mathrm{~s}, 3 \mathrm{H}), 1.61(\mathrm{~m}, 2 \mathrm{H}), 1.28(\mathrm{~m}, 4 \mathrm{H})$, $0.86(\mathrm{~m}, 3 \mathrm{H}) ;{ }^{13} \mathrm{C}-\mathrm{NMR}\left(100 \mathrm{MHz}, \mathrm{CDCl}_{3}\right) \delta: 172.5,170.0,146.4,146.3,145.9,129.4,126.5,114.0$, 107.3, 105.0, 101.0, 70.6, 69.2, 61.2, 56.9, 53.6, 40.5, 34.3, 31.2, 28.7, 24.6, 22.3, 21.0, 14.0; ESI-MS: $m / z 428[\mathrm{M}+\mathrm{H}]^{+}$; HRMS: $m / z$ calcd. for $\mathrm{C}_{24} \mathrm{H}_{30} \mathrm{NO}_{6} 428.2073[\mathrm{M}+\mathrm{H}]^{+}$; found: 428.2067 .

(1S,2S,3a $\left.a^{1} S, 12 b S\right)$-1-Acetoxy-2,3a $a^{1}, 4,5,7,12 b$-hexahydro-1H-[1,3]dioxolo[4,5-j]pyrrolo[3,2,1-de]phenanthridin-2-yl pivalate (1-acetyl-2-pivaloyllycorine, 7c). Following the previously described procedure, $95 \mathrm{mg}(0.28 \mathrm{mmol})$ of 3 gave $7 \mathrm{c}$ as a pale solid $(64 \mathrm{mg}, 56 \%) .{ }^{1} \mathrm{H}-\mathrm{NMR}\left(400 \mathrm{MHz}, \mathrm{CDCl}_{3}\right)$ $\delta: 6.77(\mathrm{~s}, 1 \mathrm{H}), 6.59(\mathrm{~s}, 1 \mathrm{H}), 5.93(\mathrm{~s}, 2 \mathrm{H}), 5.72(\mathrm{~s}, 1 \mathrm{H}), 5.52(\mathrm{~s}, 1 \mathrm{H}), 5.24(\mathrm{~s}, 1 \mathrm{H}), 4.19(\mathrm{~d}, J=14.2 \mathrm{~Hz}$, $1 \mathrm{H}), 3.56(\mathrm{~d}, J=14.1 \mathrm{~Hz}, 1 \mathrm{H}), 3.41(\mathrm{dt}, J=9.0,4.7 \mathrm{~Hz}, 1 \mathrm{H}), 2.90(\mathrm{~d}, J=10.4 \mathrm{~Hz}, 1 \mathrm{H}), 2.81(\mathrm{~d}$, $J=10.6 \mathrm{~Hz}, 1 \mathrm{H}), 2.68(\mathrm{~s}, 2 \mathrm{H}), 2.44(\mathrm{~m}, 1 \mathrm{H}), 1.97(\mathrm{~s}, 3 \mathrm{H}), 1.22(\mathrm{~s}, 9 \mathrm{H}) ;{ }^{13} \mathrm{C}-\mathrm{NMR}\left(100 \mathrm{MHz}, \mathrm{CDCl}_{3}\right)$ $\delta: 177.2,169.9,146.5,146.3,145.8,129.3,126.6,113.9,107.3,105.1,101.0,70.6,69.1,61.3,56.8$, 53.6, 40.6, 38.7, 28.6, 27.1, 20.9; ESI-MS: $m / z 414[\mathrm{M}+\mathrm{H}]^{+}$; HRMS: $m / z$ calcd. for $\mathrm{C}_{23} \mathrm{H}_{28} \mathrm{NO}_{6}$ 414.1917 [M+ H] $]^{+}$; found: 414.1912.

4-(((1S,2S,3a $\left.a^{1} S, 12 b S\right)-1$-Acetoxy-2,3a $a^{1}$ 4,5,7,12b-hexahydro-1H-[1,3]dioxolo[4,5-j]pyrrolo[3,2,1-de]phenanthridin-2-yl)oxy)-4-oxobutanoic acid (1-acetyl-2-(4-carboxyl-propionate)lycorine, 7d). Following the previously described procedure, $150 \mathrm{mg}(0.46 \mathrm{mmol})$ of $\mathbf{3}$ gave $7 \mathbf{d}$ as a white solid (178mg, 90.5\%). ${ }^{1} \mathrm{H}-\mathrm{NMR}\left(400 \mathrm{MHz}, \mathrm{CDCl}_{3}\right) \delta: 6.72(\mathrm{~s}, 1 \mathrm{H}), 6.62(\mathrm{~s}, 1 \mathrm{H}), 5.92(\mathrm{~s}, 2 \mathrm{H}), 5.76(\mathrm{~s}, 1 \mathrm{H})$, $5.58(\mathrm{~s}, 1 \mathrm{H}), 5.27(\mathrm{~s}, 1 \mathrm{H}), 4.19(\mathrm{~d}, J=14.0 \mathrm{~Hz}, 1 \mathrm{H}), 3.81(\mathrm{~d}, J=14.0 \mathrm{~Hz}, 1 \mathrm{H}), 3.50$ (brs, 1H), 3.21 (d, $J=10.8 \mathrm{~Hz}, 1 \mathrm{H}), 3.02(\mathrm{~d}, J=10.4 \mathrm{~Hz}, 1 \mathrm{H}), 2.80(\mathrm{~m}, 1 \mathrm{H}), 2.74(\mathrm{~m}, 2 \mathrm{H}), 2.54-2.60(\mathrm{~m}, 4 \mathrm{H}), 1.96$ (s, $3 \mathrm{H}) ;{ }^{13} \mathrm{C}-\mathrm{NMR}\left(100 \mathrm{MHz}, \mathrm{CDCl}_{3}\right) \delta: 175.7,171.3,169.8,147.1,146.7,143.7,127.4,126.4,115.2$, 
107.6, 105.0, 101.2, 70.0, 68.4, 60.7, 55.0, 53.2, 38.5, 29.9, 29.8, 28.7, 20.9; ESI-MS: $\mathrm{m} / z$ $[\mathrm{M}-\mathrm{H}]^{-}$; HRMS: $m / z$ calcd. for $\mathrm{C}_{22} \mathrm{H}_{24} \mathrm{NO}_{8} 430.1502[\mathrm{M}+\mathrm{H}]^{+}$; found: 430.1489 .

(1S,2S,3a $S, 12 b S)$-1-Acetoxy-2,3 $a^{1}, 4,5,7,12 b$-hexahydro-1H-[1,3]dioxolo[4,5-j]pyrrolo[3,2,1de]phenanthridin-2-yl 4-azidobenzoate (1-acetyl-2-(4-azidobenzoyl)lycorine, 7e). Following the previously described procedure described above, $115 \mathrm{mg}(0.35 \mathrm{mmol})$ of $\mathbf{3}$ gave $7 \mathrm{e}$ as a white solid $(120 \mathrm{mg}, 72.0 \%)$. ${ }^{1} \mathrm{H}-\mathrm{NMR}\left(400 \mathrm{MHz}, \mathrm{CDCl}_{3}\right) \delta: 8.02(\mathrm{~d}, J=6.8,2 \mathrm{H}), 7.02(\mathrm{~d}, J=6.8,2 \mathrm{H}), 6.77(\mathrm{~s}, 1 \mathrm{H}), 6.63(\mathrm{~s}, 1 \mathrm{H})$, $5.92(\mathrm{~s}, 1 \mathrm{H}), 5.91(\mathrm{~s}, 2 \mathrm{H}), 5.68(\mathrm{~s}, 1 \mathrm{H}), 5.53(\mathrm{~s}, 1 \mathrm{H}), 4.23(\mathrm{~d}, J=14.0,1 \mathrm{H}), 3.72(\mathrm{~d}, J=14.0,1 \mathrm{H}), 3.47(\mathrm{~m}$, $1 \mathrm{H}), 3.14(\mathrm{~d}, J=10.8,1 \mathrm{H}), 3.09(\mathrm{~d}, J=10.8,1 \mathrm{H}), 2.74(\mathrm{~m}, 2 \mathrm{H}), 2.68(\mathrm{~m}, 1 \mathrm{H}), 2.00(\mathrm{~s}, 3 \mathrm{H}) ;{ }^{13} \mathrm{C}-\mathrm{NMR}$ $\left(100 \mathrm{MHz}, \mathrm{CDCl}_{3}\right) \delta: 170.0,164.4,146.8,146.6,145.4,145.0,144.3,131.7,128.5,126.5,126.3$, $118.8,118.6,114.5,107.5,105.0,101.1,70.9,68.9,61.0,56.0,53.5,39.7,28.8,21.0$; ESI-MS: $\mathrm{m} / z$ $475[\mathrm{M}+\mathrm{H}]^{+}$; HRMS: $m / z$ calcd. for $\mathrm{C}_{25} \mathrm{H}_{23} \mathrm{~N}_{4} \mathrm{O}_{6} 475.1612[\mathrm{M}+\mathrm{H}]^{+}$; found: 475.1610 .

(1aR,2a $\left.a^{1} S, 11 b S, 11 c S\right)-2 a^{1}, 3,4,6,11 b, 11 c-H e x a h y d r o-1 a H-[1,3]$ dioxolo[4,5-j]oxireno[2,3-a]pyrrolo-

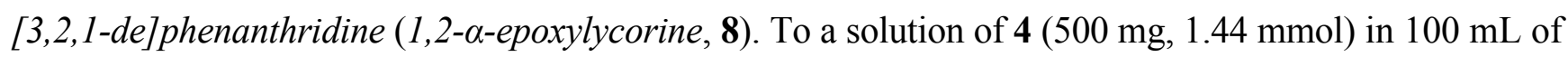
$\mathrm{CH}_{3} \mathrm{OH}$ was added $1.0 \mathrm{~g}$ of $\mathrm{CH}_{3} \mathrm{ONa}$, and the mixture was stirred in ice bath for $20 \mathrm{~min}$. DCM and water was added, and the organic layer was dried over anhydrous $\mathrm{Na}_{2} \mathrm{SO}_{4}$. After filtration, the filtrate was concentrated under reduced pressure and the crude residue was purified using a silica gel chromatography eluted with EtOAc to give 8 as a white solid (166 mg, 42.9\%). ${ }^{1} \mathrm{H}-\mathrm{NMR}$ (400 MHz, $\left.\mathrm{CDCl}_{3}\right) \delta: 7.04(\mathrm{~s}, 1 \mathrm{H}), 6.60(\mathrm{~s}, 1 \mathrm{H}), 5.94(\mathrm{~d}, J=4.4 \mathrm{~Hz}, 2 \mathrm{H}), 5.76(\mathrm{~d}, J=1.6 \mathrm{~Hz}, 1 \mathrm{H}), 4.08(\mathrm{~d}$, $J=14.0 \mathrm{~Hz}, 1 \mathrm{H}), 3.96(\mathrm{~d}, J=4.0 \mathrm{~Hz}, 1 \mathrm{H}), 3.58(\mathrm{~d}, J=14.0 \mathrm{~Hz}, 1 \mathrm{H}), 3.51(\mathrm{t}, J=4.0 \mathrm{~Hz}, 1 \mathrm{H}), 3.21(\mathrm{~m}$, $1 \mathrm{H}), 2.80(\mathrm{t}, J=13.6 \mathrm{~Hz}, 2 \mathrm{H}), 2.61(\mathrm{~m}, 1 \mathrm{H}), 2.43(\mathrm{~m}, 1 \mathrm{H}) ;{ }^{13} \mathrm{C}-\mathrm{NMR}\left(100 \mathrm{MHz}, \mathrm{CDCl}_{3}\right) \delta: 147.9$, 146.4, 146.2, 129.5, 129.2, 112.6, 107.5, 105.4, 101.0, 63.1, 57.0, 54.5, 54.0, 49.5, 40.8, 29.3; ESI-MS: $m / z 270[\mathrm{M}+\mathrm{H}]^{+}$; HRMS: $m / z$ calcd. for $\mathrm{C}_{16} \mathrm{H}_{16} \mathrm{NO}_{3} 270.1125[\mathrm{M}+\mathrm{H}]^{+}$; found: 270.1130 .

\subsubsection{General Procedure for the Preparation of 9}

Compound 8 was dissolved in amine $(5 \mathrm{~mL}$, piperidine, diethylamine, pyrrole, $n$-butylamine, isopropylamine for $\mathbf{9 a - e}$, respectively), and the solution was heated to reflux overnight. The amine was removed under reduced pressure and the crude residue was purified using silica gel chromatography to give the products.

(1S,2S,3a $\left.a^{1} S, 12 b S\right)$-2-(Piperidin-1-yl)-2,3a , 4,5,7,12b-hexahydro-1H-[1,3]dioxolo[4,5-j]pyrrolo[3,2,1de]phenanthridin-1-ol (2-piperidinelycorine, 9a). Following the previously described procedure, $175 \mathrm{mg}(0.65 \mathrm{mmol})$ of $\mathbf{8}$ gave $\mathbf{9 a}$ as a white solid $(102 \mathrm{mg}, 44.0 \%) .{ }^{1} \mathrm{H}-\mathrm{NMR}\left(400 \mathrm{MHz}, \mathrm{CDCl}_{3}\right) \delta$ : $6.89(\mathrm{~s}, 1 \mathrm{H}), 6.61(\mathrm{~s}, 1 \mathrm{H}), 5.93(\mathrm{~d}, J=4.8 \mathrm{~Hz}, 2 \mathrm{H}), 5.47(\mathrm{~m}, 1 \mathrm{H}), 4.73(\mathrm{~d}, J=2.0 \mathrm{~Hz}, 1 \mathrm{H}), 4.13(\mathrm{~d}$, $J=14.0 \mathrm{~Hz}, 1 \mathrm{H}), 3.52(\mathrm{~d}, J=14.0 \mathrm{~Hz}, 1 \mathrm{H}), 3.32-3.36(\mathrm{~m}, 2 \mathrm{H}), 2.70-2.74(\mathrm{~m}, 2 \mathrm{H}), 2.62-2.65(\mathrm{~m}, 3 \mathrm{H})$, 2.47-2.50 (m, 3H), $2.37(\mathrm{~m}, 2 \mathrm{H}), 1.55-1.59(\mathrm{~m}, 4 \mathrm{H}), 1.45(\mathrm{~m}, 2 \mathrm{H}) ;{ }^{13} \mathrm{C}-\mathrm{NMR}\left(100 \mathrm{MHz}, \mathrm{CDCl}_{3}\right) \delta$ : 146.6, 146.3, 142.3, 130.4, 127.5, 117.8, 107.9, 104.8, 101.0, 69.1, 65.2, 60.7, 56.9, 53.7, 50.6, 44.4, 28.7, 26.6, 24.5; ESI-MS: $m / z 355[\mathrm{M}+\mathrm{H}]^{+}$; HRMS: $m / z$ calcd. for $\mathrm{C}_{21} \mathrm{H}_{27} \mathrm{~N}_{2} \mathrm{O}_{3} 355.2016[\mathrm{M}+\mathrm{H}]^{+}$; found: 355.2015 . 
(1S,2S,3a $\left.a^{1} S, 12 b S\right)$-2-(Diethylamino)-2,3a $a^{1}$ 4,5, 7,12b-hexahydro-1H-[1,3]dioxolo[4,5-j]pyrrolo[3,2,1de]phenanthridin-1-ol (2-diethylaminelycorine, 9b). Following the previously described procedure, $200 \mathrm{mg}(0.74 \mathrm{mmol})$ of $\mathbf{8}$ gave $\mathbf{9 b}$ as a gray solid $(143 \mathrm{mg}, 56.5 \%) .{ }^{1} \mathrm{H}-\mathrm{NMR}\left(400 \mathrm{MHz}, \mathrm{CDCl}_{3}\right) \delta$ : $6.88(\mathrm{~s}, 1 \mathrm{H}), 6.59(\mathrm{~s}, 1 \mathrm{H}), 5.93(\mathrm{~d}, J=11.2 \mathrm{~Hz}, 2 \mathrm{H}), 5.45(\mathrm{~s}, 1 \mathrm{H}), 4.83(\mathrm{~s}, 1 \mathrm{H}), 4.13(\mathrm{~d}, J=14.0 \mathrm{~Hz}$, 1H), $3.91(\mathrm{~s}, 1 \mathrm{H}), 3.58(\mathrm{~d}, J=14.0 \mathrm{~Hz}, 1 \mathrm{H}), 3.36(\mathrm{~m}, 1 \mathrm{H}), 2.80(\mathrm{~m}, 5 \mathrm{H}), 2.67$ (brs, 2H), 2.55-2.38 (m, 2H), $1.25(\mathrm{t}, J=7.1 \mathrm{~Hz}, 6 \mathrm{H}) ;{ }^{13} \mathrm{C}-\mathrm{NMR}\left(100 \mathrm{MHz}, \mathrm{CDCl}_{3}\right) \delta: 146.6,146.3,145.8,129.7,127.1,113.0$, 107.5, 105.5, 101.0, 66.8, 65.1, 60.6, 56.5, 53.4, 45.0, 43.9, 29.0, 12.3; ESI-MS: $m / z 343[\mathrm{M}+\mathrm{H}]^{+}$; HRMS: $m / z$ calcd. for $\mathrm{C}_{20} \mathrm{H}_{27} \mathrm{~N}_{2} \mathrm{O}_{3} 343.2016[\mathrm{M}+\mathrm{H}]^{+}$; found:343.2016.

(1S,2S,3a $S, 12 b S)$-2-(1H-Pyrrol-1-yl)-2,3a ,4,5, 7,12b-hexahydro-1H-[1,3]dioxolo[4,5-j]pyrrolo[3,2,1de]phenanthridin-1-ol (2-pyrrolelycorine, 9c). Following the previously described procedure, $200 \mathrm{mg}$ $(0.74 \mathrm{mmol})$ of 8 gave $9 \mathrm{c}$ as a gray solid $(161 \mathrm{mg}, 65.3 \%) .{ }^{1} \mathrm{H}-\mathrm{NMR}\left(400 \mathrm{MHz}, \mathrm{CDCl}_{3}\right) \delta: 6.62(\mathrm{~s}$, $1 \mathrm{H}), 6.58(\mathrm{~s}, 1 \mathrm{H}), 6.56(\mathrm{~s}, 1 \mathrm{H}), 6.10(\mathrm{~s}, 1 \mathrm{H}), 5.98(\mathrm{~s}, 1 \mathrm{H}), 5.88(\mathrm{~d}, J=5.2 \mathrm{~Hz}, 2 \mathrm{H}), 5.50(\mathrm{~s}, 1 \mathrm{H}), 4.49$ $(\mathrm{s}, 1 \mathrm{H}), 4.15(\mathrm{~d}, J=14.9 \mathrm{~Hz}, 1 \mathrm{H}), 3.77(\mathrm{~s}, 1 \mathrm{H}), 3.51$ (d, $J=15.2 \mathrm{~Hz}, 1 \mathrm{H}), 3.35-3.27(\mathrm{~m}, 1 \mathrm{H}), 2.88(\mathrm{~d}$, $J=10.6 \mathrm{~Hz}, 1 \mathrm{H}), 2.77$ (d, $J=10.6 \mathrm{~Hz}, 1 \mathrm{H}), 2.67(\mathrm{~m}, 2 \mathrm{H}), 2.44-2.32(\mathrm{~m}, 1 \mathrm{H}) ;{ }^{13} \mathrm{C}-\mathrm{NMR}(100 \mathrm{MHz}$, $\left.\mathrm{CDCl}_{3}\right) \delta: 146.6,146.3,139.8,132.5,127.8,117.2,116.6,108.3,107.6,106.0,104.8,101.0,71.8$, 61.1, 57.0, 53.9, 45.3, 41.0, 28.5; ESI-MS: $m / z 337[\mathrm{M}+\mathrm{H}]^{+}$; HRMS: $m / z$ calcd. for $\mathrm{C}_{20} \mathrm{H}_{21} \mathrm{~N}_{2} \mathrm{O}_{3}$ $337.1547[\mathrm{M}+\mathrm{H}]^{+}$; found:337.1543.

(1S,2S,3a $\left.a^{1} S, 12 b S\right)$-2-(Butylamino)-2,3a , 4,5, 7,12b-hexahydro-1H-[1,3]dioxolo[4,5-j]pyrrolo[3,2,1de]phenanthridin-1-ol (2-butylaminelycorine, 9d). Following the previously described procedure described above, $143 \mathrm{mg}(0.36 \mathrm{mmol})$ of $\mathbf{8}$ gave $9 \mathbf{d}$ as a pale yellow solid $(132 \mathrm{mg}, 72.8 \%)$. ${ }^{1} \mathrm{H}-\mathrm{NMR}$ $\left(400 \mathrm{MHz} \mathrm{CDCl}_{3}\right) \delta: 6.85(\mathrm{~s}, 1 \mathrm{H}), 6.58(\mathrm{~s}, 1 \mathrm{H}), 5.92(\mathrm{~d}, J=5.6 \mathrm{~Hz}, 2 \mathrm{H}), 5.48(\mathrm{~s}, 1 \mathrm{H}), 4.50(\mathrm{~s}, 1 \mathrm{H})$, $4.13(\mathrm{~d}, J=14.4 \mathrm{~Hz}, 1 \mathrm{H}), 3.48(\mathrm{~d}, J=14.0 \mathrm{~Hz}, 1 \mathrm{H}), 3.33(\mathrm{~m}, 1 \mathrm{H}), 3.24(\mathrm{~s}, 1 \mathrm{H}), 2.74(\mathrm{~m}, 3 \mathrm{H}), 2.60(\mathrm{~m}$, $3 \mathrm{H}), 2.32(\mathrm{~m}, 1 \mathrm{H}), 1.44(\mathrm{~m}, 2 \mathrm{H}), 1.34(\mathrm{~m}, 2 \mathrm{H}), 0.92(\mathrm{t}, J=7.2 \mathrm{~Hz}, 3 \mathrm{H}) ;{ }^{13} \mathrm{C}-\mathrm{NMR}\left(100 \mathrm{MHz}, \mathrm{CDCl}_{3}\right)$ $\delta: 146.5,146.2,141.5,130.4,128.0,117.6,107.7,104.7,100.9,69.8,61.6,61.2,57.2,53.9,48.3,41.7$, 32.6, 28.6, 20.4, 14.0; ESI-MS: $m / z 343[\mathrm{M}+\mathrm{H}]^{+}$; HRMS: $m / z$ calcd. for $\mathrm{C}_{20} \mathrm{H}_{27} \mathrm{~N}_{2} \mathrm{O}_{3} 343.2016[\mathrm{M}+\mathrm{H}]^{+}$; found: 343.2022 .

(1S,2S,3a $\left.a^{1} S, 12 b S\right)$-2-(Isopropylamino)-2,3a $, 4,5,7,12$ b-hexahydro-1H-[1,3]dioxolo[4,5-j]pyrrolo[3,2,1-de]phenanthridin-1-ol (2-isopropylaminelycorine, 9e). Following the previously described procedure, $160 \mathrm{mg}(0.59 \mathrm{mmol})$ of $\mathbf{8}$ gave $9 \mathrm{e}$ as a pale yellow solid (175 $\mathrm{mg}, 90.4 \%)$. ${ }^{1} \mathrm{H}-\mathrm{NMR}\left(400 \mathrm{MHz}, \mathrm{CDCl}_{3}\right) \delta: 6.85(\mathrm{~s}, 1 \mathrm{H}), 6.57(\mathrm{~s}, 1 \mathrm{H}), 5.91(\mathrm{~d}, J=5.2 \mathrm{~Hz}, 2 \mathrm{H}), 5.45(\mathrm{~s}, 1 \mathrm{H}), 4.45$ $(\mathrm{s}, 1 \mathrm{H}), 4.12(\mathrm{~d}, J=14.4 \mathrm{~Hz}, 1 \mathrm{H}), 3.48(\mathrm{~d}, J=14.0 \mathrm{~Hz}, 1 \mathrm{H}), 3.31(\mathrm{~m}, 1 \mathrm{H}), 3.29(\mathrm{~s}, 1 \mathrm{H}), 3.01(\mathrm{~m}, 1 \mathrm{H})$, $2.72(\mathrm{~d}, J=10.8 \mathrm{~Hz}, 1 \mathrm{H}), 2.60(\mathrm{~d}, J=10.8 \mathrm{~Hz}, 1 \mathrm{H}), 2.58(\mathrm{~m}, 2 \mathrm{H}), 2.31(\mathrm{~m}, 1 \mathrm{H}), 1.08(\mathrm{~s}, 3 \mathrm{H}), 1.06$ (s, $3 \mathrm{H}) ;{ }^{13} \mathrm{C}-\mathrm{NMR}\left(100 \mathrm{MHz}, \mathrm{CDCl}_{3}\right) \delta: 145.5,145.1,139.9,129.3,127.1,116.8,106.6,103.7,99.9$, 69.8, 60.1, 57.7, 56.2, 52.9, 46.4, 40.4, 27.6, 22.5, 22.2; ESI-MS: $m / z 329$ [M+ H] ; HRMS: $m / z$ calcd. for $\mathrm{C}_{19} \mathrm{H}_{25} \mathrm{~N}_{2} \mathrm{O}_{3} 329.1860[\mathrm{M}+\mathrm{H}]^{+}$; found: 329.1855 .

\section{Conclusions}

A series of novel lycorine derivatives were synthesized and their in vitro anticancer activities were evaluated. Diverse amine substituents were introduced into the C-2 position of lycorine for the first 
time and this series of derivatives exhibited good anticancer properties towards seven cell lines. This provides new opportunities for the investigation of novel lycorine derivatives as anticancer agents. Interestingly, selectivity towards different cell lines with this series of lycorine derivatives was observed. Compounds with different selectivity profiles could potentially be used as probes to study the differences between cell lines.

\section{Acknowledgments}

This work was supported by the National Natural Science Foundation of China (Grant No. 21202087), the National Basic Research Program of China (973 program, Grant No. 2013CB911104), the Fundamental Research Funds for the Central Universities (Grant No. 65124002), the Specialized Research Fund for the Doctoral Program of Higher Education Ministry of Education of China (Grant No. 20120031120049), the Tianjin Science and Technology Program (Grant No. 13JCYBJC24300, 13JCQNJC13100), the Scientific Research Starting Foundation of Returned Overseas Chinese Scholars, Ministry of Education of China and the "111" Project of the Ministry of Education of China (Project No. B06005). Sincere thanks to Alex Santayana for English corrections.

\section{Conflicts of Interest}

The authors declare no conflict of interest.

\section{References}

1. Patil, S.D.; Chaudhari, M.A.; Sapkale, P.V.; Chaudhari, R.B. A recent review on anticancer herbal drugs. J. Drug Discov. Ther. 2013, 1, 77-84.

2. Viswanath, A.N.I.; Pae, A.N. Resistance by Allostery: A Novel perspective for Eg5-targeted drug design. J. Med. Chem. 2013, 56, 6314-6316.

3. Newman, D.J.; Cragg, G.M. Natural products as sources of new drugs over the 30 years from 1981 to 2010. J. Nat. Prod. 2012, 75, 311-335.

4. Wu, S.B.; Pang, F.; Wen, Y.; Zhang, H.-F.; Zhao, Z.; Hu, J.F. Antiproliferative and apoptotic activities of linear Furocoumarins from Notopterygium incisum on cancer cell lines. Planta Med. 2010, 76, 82-85.

5. Evidente, A.; Kornienko, A. Anticancer evaluation of structurally diverse Amaryllidaceae alkaloids and their synthetic derivatives. Phytochem. Rev. 2009, 8, 449-459.

6. Kornienko, A.; Evidente, A. Chemistry, biology, and medicinal potential of Narciclasine and its congeners. Chem. Rev. 2008, 108, 1982-2014.

7. Liu, X.S.; Jiang, J.; Jiao, X.Y.; Wu, Y.E.; Lin, J.H.; Cai, Y.M. Lycorine induces apoptosis and down-regulation of Mcl-1 in human leukemia cells. Cancer Lett. 2009, 274, 16-24.

8. Jokhadze, M.; Eristavi, L.; Kutchukhidze, J.; Chariot, A.; Angenot, L.; Tits, M.; Jansen, O.; Frédérich, M. In vitro cytotoxicity of some medicinal plants from Georgian Amaryllidaceae. Phytother. Res. 2007, 21, 622-624.

9. Li, Y.; Liu, J.; Tang, L.J.; Shi, Y.W.; Ren, W.; Hu, W.X. Apoptosis induced by lycorine in KM3 cells is associated with the G0/G1 cell cycle arrest. Oncol. Rep. 2007, 17, 377-384. 
10. Campbell, W.E.; Nair, J.J.; Gammon, D.W.; Codina, C.; Bastida, J.; Viladomat, F.; Smith, P.J.; Albrecht, C.F. Bioactive alkaloids from Brunsvigia radulosa. Phytochemistry 2000, 53, 587-591.

11. McNulty, J.; Nair, J.J.; Bastida, J.; Pandey, S.; Griffin, C. Structure-activity studies on the lycorine pharmacophore: A potent inducer of apoptosis in human leukemia cells. Phytochemistry 2009, 70, 913-919.

12. Lamoral-Theys, D.; Andolfi, A.; Van Goietsenoven, G.; Cimmino, A.; Le Calve, B.; Wauthoz, N.; Megalizzi, V.; Gras, T.; Bruyere, C.; Dubois, J.; et al. Lycorine, the main phenanthridine Amaryllidaceae alkaloid, exhibits significant antitumor activity in cancer cells that display resistance to proapoptotic stimuli: An investigation of structure-activity relationship and mechanistic insight. J. Med. Chem. 2009, 52, 6244-6256.

13. Evdokimov, N.M.; Lamoral-Theys, D.; Mathieu, V.; Andolfi, A.; Frolova, L.V.; Pelly, S.C.; van Otterlo, W.A.L.; Magedov, I.V.; Kiss, R.; Evidente, A.; et al. In search of a cytostatic agent derived from the alkaloid lycorine: Synthesis and growth inhibitory properties of lycorine derivatives. Bioorg. Med. Chem. 2011, 19, 7252-7261.

14. Liu, J.; Hu, W.-X.; He, L.-F.; Ye, M.; Li, Y. Effects of lycorine on HL-60 cells via arresting cell cycle and inducing apoptosis. FEBS Lett. 2004, 578, 245-250.

15. Li, L.; Dai, H.-J.; Ye, M.; Wang, S.-L.; Xiao, X.-J.; Zheng, J.; Chen, H.-Y.; Luo, Y.-H.; Liu, J. Lycorine induces cell-cycle arrest in the G0/G1 phase in K562 cells via HDAC inhibition. Cancer Cell Int. 2012, 12, 49.

16. Cao, Z.; Yu, D.; Fu, S.; Zhang, G.; Pan, Y.; Bao, M.; Tu, J.; Shang, B.; Guo, P.; Yang, P.; et al. Lycorine hydrochloride selectively inhibits human ovarian cancer cell proliferation and tumor neovascularization with very low toxicity. Toxicol. Lett. 2013, 218, 174-185.

17. Liu, R.; Cao, Z.; Tu, J.; Pan, Y.; Shang, B.; Zhang, G.; Bao, M.; Zhang, S.; Yang, P.; Zhou, Q. Lycorine hydrochloride inhibits metastatic melanoma cell-dominant vasculogenic mimicry. Pigm. Cell Melanoma Res. 2012, 25, 630-638.

18. Wildman, W.C.; Heimer, N.E. Alkaloid biosynthesis and interconversions. Conversion of caranine to lycorine. J. Am. Chem. Soc. 1967, 89, 5265-5269.

19. Wu, S.B.; Su, J.J.; Sun, L.H.; Wang, W.X.; Zhao, Y.; Li, H.; Zhang, S.P.; Dai, G.H; Wang, C.G.; $\mathrm{Hu}$, J.F. Triterpenoids and steroids from the fruits of Melia toosendan and their cytotoxic effects on two human cancer cell lines. J. Nat. Prod. 2010, 73, 1898-1906.

20. Dasari, R.; Banuls, L.M.Y.; Masi, M.; Pelly, S.C.; Mathieu, V.; Green, I.R.; van Otterlo, W.A.L.; Evidente, A.; Kiss, R.; Kornienko, A. C1,C2-ether derivatives of the Amaryllidaceae alkaloid lycorine: Retention of activity of highly lipophilic analogues against cancer cells. Bioorg. Med. Chem. Lett. 2014, 24, 923-927.

Sample Availability: Samples of compounds 1-9 are available from the authors.

(C) 2014 by the authors; licensee MDPI, Basel, Switzerland. This article is an open access article distributed under the terms and conditions of the Creative Commons Attribution license (http://creativecommons.org/licenses/by/3.0/). 